\title{
Supersymmetric spin-phonon coupling prevents odd integer spins from quantum tunneling
}

\author{
Kilian Irländer ${ }^{1}\left(\mathbb{D}\right.$, Heinz-Jürgen Schmidt ${ }^{2} \mathbb{C}$, and Jürgen Schnack ${ }^{1, a}{ }^{(\mathbb{D}}$ \\ 1 Fakultät für Physik, Universität Bielefeld, Postfach 100131, 33501 Bielefeld, Germany \\ 2 Fachbereich Physik, Universität Osnabrück, 49069 Osnabrück, Germany
}

Received 27 January 2021 / Accepted 27 February 2021 / Published online 23 March 2021 (C) The Author(s) 2021

\begin{abstract}
Quantum tunneling of the magnetization is a phenomenon that impedes the use of small anisotropic spin systems for storage purposes even at the lowest temperatures. Phonons, usually considered for temperature dependent relaxation of magnetization over the anisotropy barrier, also contribute to magnetization tunneling for integer spin quantum numbers. Here we demonstrate that certain spin-phonon Hamiltonians are unexpectedly robust against the opening of a tunneling gap, even for strong spin-phonon coupling. The key to understanding this phenomenon is provided by an underlying supersymmetry that involves both spin and phonon degrees of freedom.
\end{abstract}

\section{Introduction}

Single-ion magnetic anisotropy provides the simplest mechanism for fundamental phenomena such as magnetic bistability as well as quantum tunneling of the magnetization [1-3]. The Hamiltonian is so simple that any student after an introductory course on quantum mechanics can diagonalize it. It consists of two terms:

$$
\begin{gathered}
\underset{\sim}{H_{\mathrm{SI}}}=D(\underset{\sim}{s} z)^{2}+E\left\{\left({\underset{\sim}{s} x}^{s}\right)^{2}-(\underset{\sim}{s} y)^{2}\right\} \\
=D(\underset{\sim}{s} z)^{2}+\frac{E}{2}\left\{\left({\underset{\sim}{s}}^{+}\right)^{2}+\left({\underset{\sim}{s}}^{-}\right)^{2}\right\},
\end{gathered}
$$

which, for obvious reasons, have been termed $D$ - and $E$-term, see, e.g., [1] for a full account of the story. A negative $D, D<0$, results in an easy-axis anisotropy which, in the absence of the $E$-term, would express itself as a perfect parabolic anisotropy barrier, compare l.h.s. of Fig. 1. $E$ leads to a splitting of the otherwise degenerate pairs of levels left and right of the barrier if the considered spin is integer, compare r.h.s. of Fig. 1. If the spin is half integer, Kramers' theorem applies, and the levels are bound to be degenerate for $B=0$.

In a magnetic field applied along the easy-axis one encounters a perfect level crossing for $E=0$; such systems - single ion magnets (SIM) or single molecule magnets (SMM) - do show bistability of the magnetization and are thus suitable candidates for magnetic storage devices [5-12]. In case of a splitting of the two lowest levels, one observes an avoided level crossing as

\footnotetext{
${ }^{\mathrm{a}} \mathrm{e}-\mathrm{mail}: \quad$ jschnack@uni-bielefeld.de $\quad$ (corresponding author)
}

a function of applied field as depicted on the r.h.s. of Fig. 1. The magnetization is not bi-stable at $B=0$, instead it tunnels as described for two-level systems by Landau, Zener, and Stueckelberg [13-15]. The splitting therefore is also called tunnel splitting.

To our surprise, this simple scheme - tunnel splitting for integer spins, no tunnel splitting for half-integer spins - needs a modification for integer spins in the case of phonon-induced tunnel splitting, if the spin is coupled to phonons of the material in a certain way. It may then happen that the tunnel splitting opens up only for even spin quantum numbers, whereas one observes a perfect level crossing in the case of odd spin quantum numbers.

This behavior is exceptional for two reasons. The insight that spin-phonon interactions open a tunnel splitting dates back to the late 1960's [17-19] and was discussed in connection with molecular magnetism ever since then, see, e.g., [20-26]. Here we demonstrate with an example that special phonon modes exist that do not open up a tunneling gap, independent of the spinphonon coupling strength. The second unexpected finding is that this behavior is not rooted in the phonon subsystem alone but can be traced back to a combined symmetry of the spin and phonon modes, which resembles a supersymmetry of the problem. Note that the authors of [27] have already recognized that the rules concerning the occurrence of avoided level crossings are overridden by existing symmetries. We provide a fundamental example.

The article is organized as follows. In Sect. 2, we introduce our model and the applied method. Section 3 presents the results. After a discussion in Sect. 4 and the references an extended appendix provides more detailed insight. 

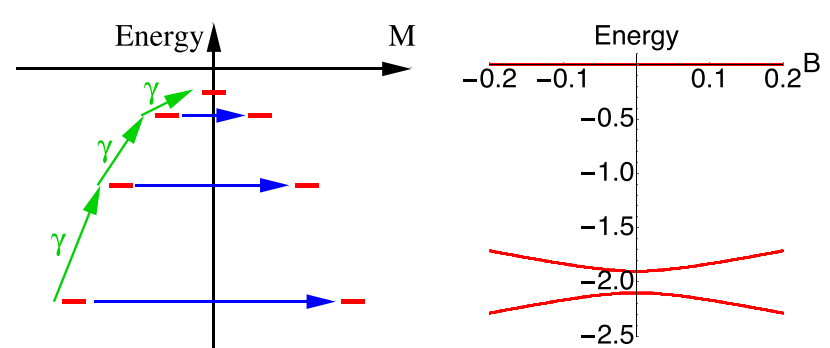

Fig. 1 L.h.s.: Sketch of the low-lying energy levels of a spin $s=3$ with dominant easy-axis anisotropy vs. magnetic quantum number. Red bars denote energy eigenvalues. Blue arrows show magnetization tunneling pathways for states with negative magnetic quantum number, and green arrows depict some of the possible excitations due to phonons, compare e.g. [4]. R.h.s.: Example of a tunnel splitting for a spin $s=1$ with $D<0$ and $E \neq 0$ anisotropy terms

\section{Model and method}

Specifically, we consider the following Hamiltonian

$$
\underset{\sim}{H}=\underset{\sim}{H} \mathrm{SI}+\underset{\sim}{H} \mathrm{HO}+\underset{\sim}{H} \text { Zeeman }
$$

where the interaction of the spin with the phonons of the system is reduced to a single harmonic oscillator:

$$
\underset{\sim}{H} \mathrm{HO}=\omega\left({\underset{\sim}{a}}_{\sim}^{\dagger} a+\frac{1}{2}\right)
$$

for educational reasons. $\underset{\sim}{a}{ }^{\dagger}$ and $\underset{\sim}{a}$ are the creation and destruction operators of a certain normal mode that couples to the spin as outlined below. The spin also interacts with the external magnetic field along the easy axis described by $\underset{\sim}{\mathrm{H}}$ Zeeman .

Key to our observation is the way the oscillator mode couples to the spin. Out of the many couplings possible $[16,21-25,28]$, we investigate those cases where the phonons modify only the E-terms, compare Fig. 2 and first term of Eq. (6) of Ref. [16] for a specific relation to the strain tensor. We assume two different couplings, a linear coupling

$$
E=\alpha_{1}\left({\underset{\sim}{a}}^{\dagger}+\underset{\sim}{a}\right)
$$

where $E$ is proportional to the generalized coordinate of the normal mode as well as a quadratic coupling

$$
E=\alpha_{2}\left(\underset{\sim}{a^{\dagger}}+\underset{\sim}{a}\right)^{2}
$$

It will later turn out that the fundamental difference we found exists between odd and even powers of the generalized coordinate $\left({\underset{\sim}{\sim}}^{\dagger}+\underset{\sim}{a}\right)$ of the normal mode.

Hamiltonian 3 can be diagonalized numerically exactly using the product basis $|m, n\rangle$, with $m$ being the magnetic quantum number and $n$ the oscillator

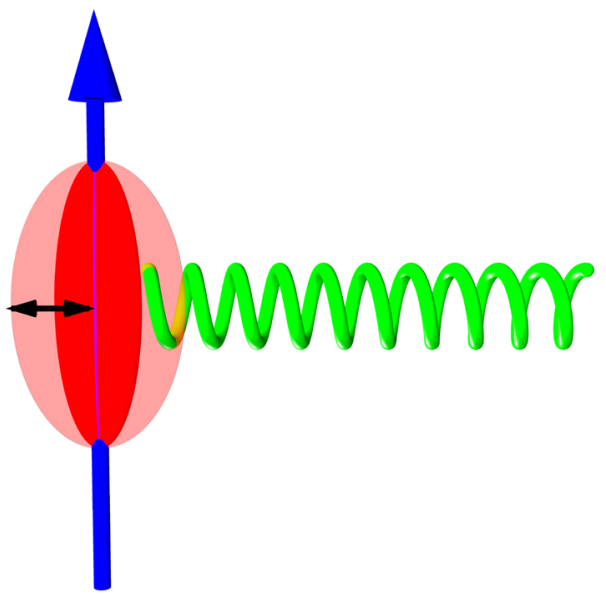

Fig. 2 Sketch of the coupling of the anisotropy tensor to phonons of the material. The reddish ellipsoid represents the anisotropy tensor whose components ( $E$-terms) along major axes perpendicular to the easy axis are modified via a coupling to a special phonon mode (green coil). For a relation to the strain tensor compare e.g. the first term of Eq. (6) of Ref. [16] for a specific example

quantum number, if $n$ is cut at some maximal value $n_{\max }$. We investigated various $n_{\max }=0,1, \ldots, 5$, and it turns out that small $n_{\max }$, even $n_{\max }=1$, are sufficient to accurately describe ground state properties $[26]$.

\section{Results}

A numerical diagonalization with practically arbitrary parameters reveals that an odd-even effect determines the tunnel splitting for the linear coupling, see Fig. 3.
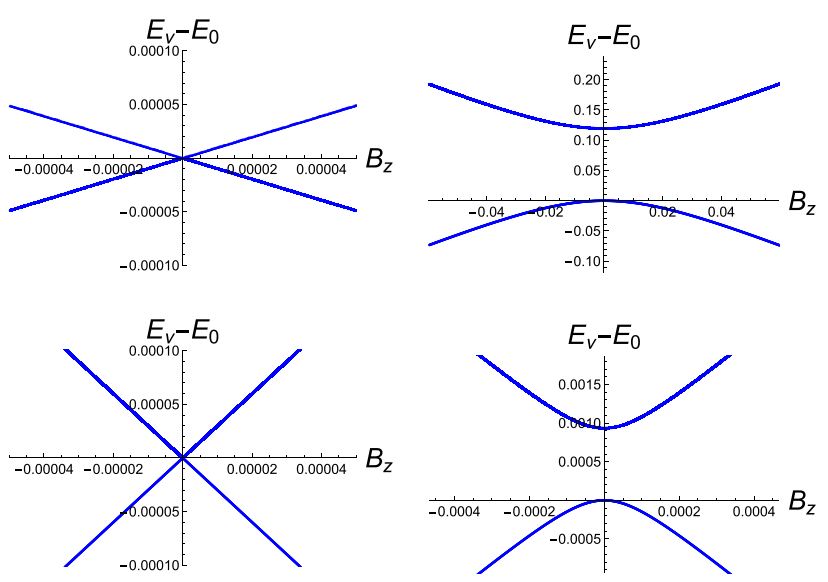

Fig. 3 Linear coupling: Lowest energy eigenvalues relative to $E_{0}$ vs. magnetic field strength for different integer spin quantum numbers $s=\{1, \ldots, 4\}$ (from left to right and top to bottom) with $D=-5, n_{\max }=1, \alpha_{1}=0.5, \omega=5$ in natural units. $E_{0}$ denotes the ground state energy at $B=0$ 

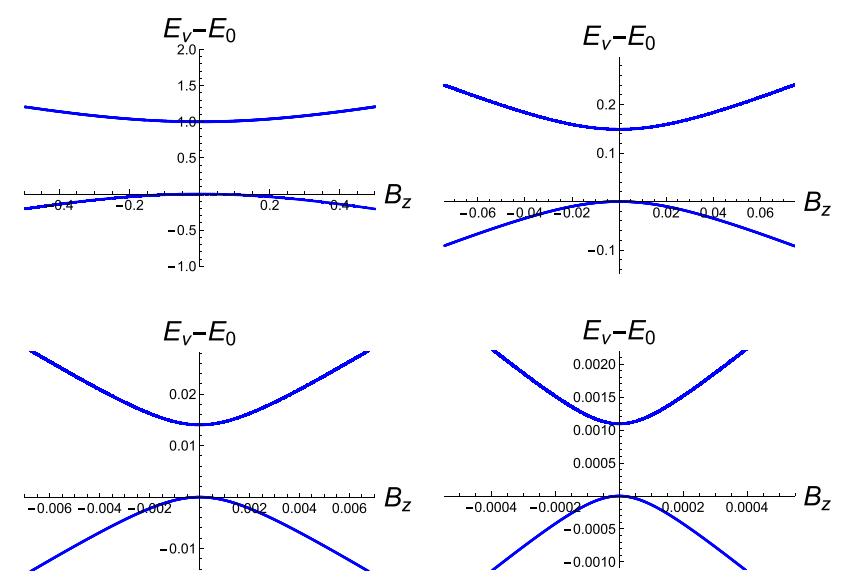

Fig. 4 Quadratic coupling: Lowest energy values relative to $E_{0}$ vs. magnetic field strength for different integer spin quantum numbers $s=\{1, \ldots, 4\}$ (from left to right and top to bottom) with $D=-5, n_{\max }=1, \alpha_{2}=0.5, \omega=5$ in natural units. $E_{0}$ denotes the ground state energy at $B=0$

For the quadratic coupling, the tunneling gap opens for all integer spins, compare Fig. 4. The behavior persists for higher spin quantum numbers and $n_{\max }$ as we have numerically verified.

The case of a quadratic coupling, or in general of a coupling with an even power of $\left({\underset{\sim}{\sim}}^{\dagger}+\underset{\sim}{a}\right)$, can be immediately understood when considering that, whatever the eigenstates of the total Hamiltonian 3, the oscillator part will contribute zero-point motion, i.e. a parameter $E$ definitely larger than zero. Similar to the case with constant $E$, these yield a tunnel splitting for all integer spin quantum numbers $[25,26]$.

The case of a linear coupling, where the unexpected level crossings for odd integer spins occur, needs a deeper investigation. The key to understanding this phenomenon is provided by an underlying not yet considered supersymmetry together with some reasonable estimates. To this end we rewrite Hamiltonian 2 using for the normal mode the coordinate operator $\xi \propto\left(\underset{\sim}{a}{ }^{\dagger}+\underset{\sim}{a}\right)$

$$
\underset{\sim}{H} \mathrm{SI}=D\left(\underset{\sim}{s_{z}}\right)^{2}+\alpha \sqrt{2 \mu \omega} \underset{\sim}{\xi}\left[(\underset{\sim}{s})^{2}-\left(\underset{\sim}{s_{y}}\right)^{2}\right],
$$

with $\mu$ being the oscillator mass ( $\hbar=1$ throughout the paper). It is now more obvious that this operator, and also the full Hamiltonian without Zeeman term, have got a fourfold symmetry with respect to the following symmetry operation

$$
\underset{\sim}{U}=\exp \left\{-i \pi \underset{\sim}{s_{z}} / 2\right\} \otimes \underset{\sim}{\prod},
$$

which inverts $\xi$ (parity operation $\underset{\sim}{\prod}$ acting on $\xi$ ) and simultaneously rotates the spin vector operator about its $z$-axis by $\pi / 2$. The cyclic group generated by $\underset{\sim}{U}$ is of order four and has got four irreducible repre-

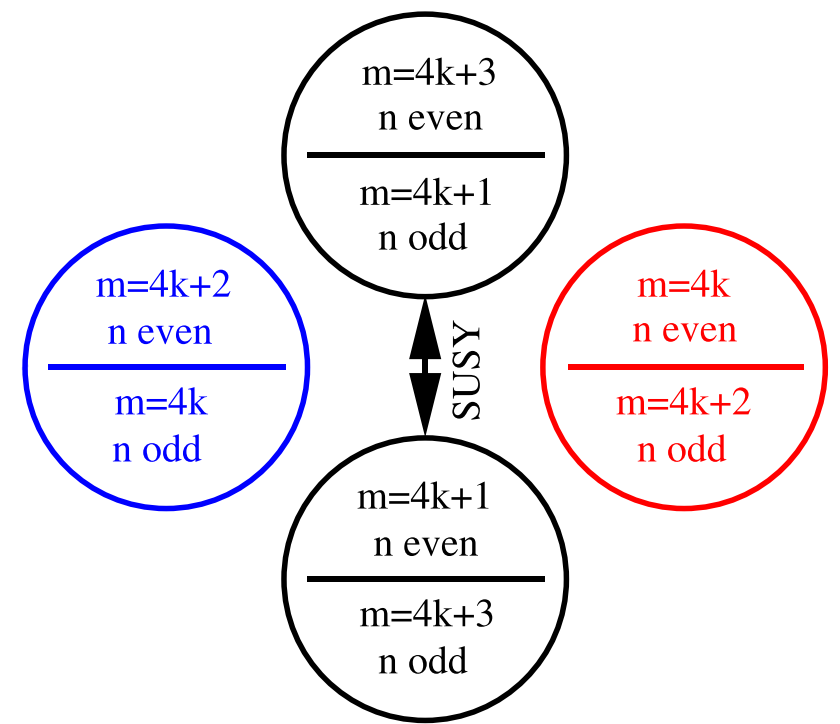

Fig. 5 Graphical representation of the four sets of product basis states spanning $\mathcal{H}_{\ell}, \ell=0,1,2,3$ (clockwise from 3 o'clock) according to their eigenvalue with respect to the symmetry transform $\underset{\sim}{U}$, see $(9) . m$ denotes the magnetic quantum number, $k$ is an integer, and $n$ is the oscillator quantum number

sentations that may be labeled by their characters $\exp \{-i \pi \ell / 2\}, \ell=0,1,2,3$. All four irreps are realized by product basis states that are already eigenstates of $\underset{\sim}{\sim}:$

$$
\begin{aligned}
\underset{\sim}{U}|m, n\rangle & =\exp \{-i \pi m / 2\}(-1)^{n}|m, n\rangle \\
& =(-i)^{m}(-1)^{n}|m, n\rangle,
\end{aligned}
$$

and can thus be grouped according to these eigenvalues. Therefore, the total Hilbert space can be decomposed into four mutually orthogonal subspaces $\mathcal{H}=$ $\mathcal{H}_{0} \oplus \mathcal{H}_{1} \oplus \mathcal{H}_{2} \oplus \mathcal{H}_{3}$. This is graphically depicted in Fig. 5 .

The system possesses a second symmetry

$$
\underset{\sim}{V}=\exp \{-i \pi \underset{\sim}{s} x\} \otimes \underset{\sim}{\mathbb{1}}
$$

which affects the spin part only. It leaves $\underset{\sim}{\underset{\sim}{S}}$ invariant and rotates $\underset{\sim}{\underset{\sim}{s}} y$ and $\underset{\sim}{\underset{\sim}{s}}$ into their respective negatives. This operation also commutes with the Hamiltonian, since $\underset{\sim}{H}$ depends on the squares of these operators. But symmetry $\underset{\sim}{V}$ does not commute with $\underset{\sim}{U}$, at least not on the full Hilbert space. However, thanks to the properties of Hamiltonian 1, basis states $|m, n\rangle$ are only connected to $|m, n\rangle$ by $(\underset{\sim}{s})^{2}$ and to $|m \pm 2, n\rangle$ by $\left({\underset{\sim}{s}}^{+}\right)^{2}$ and $(\underset{\sim}{s})^{2}$, which divides the Hilbert space into a direct sum of two mutually orthogonal subspaces for even and odd $m$, that is

$$
\begin{aligned}
\mathcal{H} & =\mathcal{H}_{\text {even }} \oplus \mathcal{H}_{\text {odd }}, \quad \text { with } \\
\mathcal{H}_{\text {even }} & =\mathcal{H}_{0} \oplus \mathcal{H}_{2},
\end{aligned}
$$




$$
\mathcal{H}_{\text {odd }}=\mathcal{H}_{1} \oplus \mathcal{H}_{3} .
$$

$\underset{\sim}{U}$ and $\underset{\sim}{V}$ commute on $\mathcal{H}_{\text {even }}$, whereas they anticommute on $\mathcal{H}_{\text {odd }}$. Using concepts from supersymmetry, where $\underset{\sim}{U}$ and $\underset{\sim}{V}$ can be embedded into a Lie superalgebra [29], one can derive the following conclusions, see also appendix.

One can show that symmetry $\underset{\sim}{V}$ maps $\mathcal{H}_{1}$ onto $\mathcal{H}_{3}$ and vice versa and eigenstates of $\underset{\sim}{H}$ that are element of one of these two subspaces onto the respective eigenstates in the other space. Therefore, their energy eigenvalues must be at least twofold degenerate. $\underset{\sim}{V}$ leaves $\mathcal{H}_{0}$ and $\mathcal{H}_{2}$ invariant. Since $\mathcal{H}_{1}$ and $\mathcal{H}_{3}$ contain the states with odd $m$ quantum number, these states are bound to be degenerate at $B=0$ and thus have to cross. Eigenstates with even values of $m$ are not degenerate by symmetry, except for a possible, but unlikely accidental degeneracy. These levels split, and therefore we observe an avoided level crossing in such cases.

Although from the point of view of applications only interesting for the ground state, this observation holds also for excited states. All levels that have been degenerate for $E=0$ split under linear coupling to phonons if $m$ is an even integer and they remain degenerate if $m$ is an odd integer.

The question whether the pair of levels that make up the ground state without anisotropy, i.e. without coupling to the phonons, remains a (tunnel-split) pair of ground state levels shall be answered using perturbation theory. If the interaction with the phonon subsystem is weak, i.e., much weaker than given by the energy scale provided by the easy-axis anisotropy $D$ - and only these systems are technologically interesting - we find that the ground states consist to a large extent of

$$
|m=s, n=0\rangle \text { and }|m=-s, n=0\rangle
$$

for odd $m$ and of the two superpositions

$$
|m=s, n=0\rangle \pm|m=-s, n=0\rangle
$$

for even $m$. Admixtures of other basis states remain very small, see analytical examples for $s=1$ and $s=2$ in the appendix. Therefore, the related energy eigenvalues of the ground states also deviate only little from those of the axial system with $E=0$. Our numerical studies for spin quantum numbers up to $s=8$ and $n_{\max }=5$, of which a part is shown in Figs. 3 and 4, arrive at the same conclusions.

\section{Discussion}

The aim of the present paper is not to solve the spinphonon problem in all details or to model specific magnetic molecules realistically. Instead, our findings provide an interesting insight into the effect of certain phonon modes on the tunneling gap at an avoided level crossing. Counter-intuitive for a physics approach, the linear term of a power series describing the interaction of the phonon mode with the $E$ term of the anisotropy tensor - which one would naively assume to have the strongest effect - does not lead to any tunnel splitting in the case of odd integer spin quantum numbers. It is the quadratic term that does the job. The symmetry argument we found holds for all odd powers of $(\underset{\sim}{a}+\underset{\sim}{a})$ where no splitting is observed for odd integer spins, whereas for all even powers thereof, a tunnel splitting exists.

Further on, the argument carries through also for coupled spins. If spins interact via a Heisenberg interaction, and if the phonons affect the anisotropy tensors as described, our findings hold for the zero-field split multiplets in case of integer total spin.

Thus, we understand from a more fundamental point of view why a phonon that tilts an anisotropy tensor, as investigated in [26], always opens a tunneling gap (for any integer spin). The tilt, expressed as changes of both $E$ and $D$, yields a Taylor series in $E$ that contains only even powers of the oscillator elongation. Thanks to the zero-point motion of the oscillator, this leads to $E>0$ and an immediate opening of the tunneling gap, as explained above. In addition, also $D$ is modified contrary to the investigation in this paper.

In a real magnetic molecule many phonon modes contribute to the physical behavior [17-25]. However, the rational design of ligands and chemical bonds aims at reducing the number of decohering and relaxing low-energy modes. It is therefore desirable to qualitatively understand the character of the remaining active modes. With this article we hope to contribute insight for a class of supersymmetric spin-phonon systems where due to an odd-even effect the impact on the tunneling gap is known a priori.

Odd-even effects appear in many places in physics. In the context of tunneling and supersymmetry, we found an article on Inelastic cotunneling into a superconductor nano particle, where odd and even numbers of tunneling electrons behaved differently [30], for curiosity.

Acknowledgements We thank Eugenio Coronado, Luis Escalera-Moreno, Stefano Sanvito, Mihail Atanasov, Nick Chilton as well as Mark Pederson for valuable discussions.

Funding Open Access funding enabled and organized by Projekt DEAL. This work was supported by the Deutsche Forschungsgemeinschaft DFG 355031190 (FOR 2692); 397300368 (SCHN 615/25-1).

Data Availability Statement This manuscript has no associated data or the data will not be deposited. [Authors' comment: This is a theoretical study, there are no experimental data has been listed.]

Open Access This article is licensed under a Creative Commons Attribution 4.0 International License, which permits use, sharing, adaptation, distribution and reproduction in 
any medium or format, as long as you give appropriate credit to the original author(s) and the source, provide a link to the Creative Commons licence, and indicate if changes were made. The images or other third party material in this article are included in the article's Creative Commons licence, unless indicated otherwise in a credit line to the material. If material is not included in the article's Creative Commons licence and your intended use is not permitted by statutory regulation or exceeds the permitted use, you will need to obtain permission directly from the copyright holder. To view a copy of this licence, visit http://creativecomm ons.org/licenses/by/4.0/.

\section{A Appendix}

In this appendix, we will substantiate the statements in the main part of the paper about the role of supersymmetry for the problem under consideration, see Sect. A.1. In the following two sections, we will further confirm the numerical results on the degeneracy of the ground state by exact analytical diagonalization of the Hamiltonian (without Zeeman term) for the case of $s=1$, see Sect. A.2, and by rigorous estimates for the analogous case of $s=2$, see Sect. A.3.

\section{A.1 Role of supersymmetry}

The even-odd effect described in the main part of the paper depends on the fact that the second symmetry:

$$
\underset{\sim}{V}=\exp \left\{-i \pi \underset{\sim}{s_{x}}\right\} \otimes \underset{\sim}{\mathbb{1}}
$$

permutes the eigenspaces $\mathcal{H}_{\ell}, \ell=0,1,2,3$, of the first symmetry

$$
\underset{\sim}{U}=\exp \{-i \pi \underset{\sim}{s} z / 2\} \otimes \underset{\sim}{\prod}
$$

in the following way:

$$
\begin{aligned}
\underset{\sim}{V} \mathcal{H}_{0} & =\mathcal{H}_{0}, \underset{\sim}{V} \mathcal{H}_{2}=\mathcal{H}_{2}, \\
\text { but } \underset{\sim}{V} \mathcal{H}_{1} & =\mathcal{H}_{3} \text { and } \underset{\sim}{V} \mathcal{H}_{3}=\mathcal{H}_{1} .
\end{aligned}
$$

This in turn follows from the observation that $\underset{\sim}{U}$ and $\underset{\sim}{V}$ commute on the subspace $\mathcal{H}_{\text {even }}=\mathcal{H}_{0} \oplus \mathcal{H}_{2}$, but anti-commute on $\mathcal{H}_{\text {odd }}=\mathcal{H}_{1} \oplus \mathcal{H}_{3}$. In fact, let $\phi \in \mathcal{H}_{\ell}, \ell=1,3$, be an eigenvector of $\underset{\sim}{U}$,

$$
\underset{\sim}{U} \phi=(-i)^{\ell} \phi
$$

then it follows that

$$
\begin{gathered}
\underset{\sim}{U}(\underset{\sim}{V} \phi)=-\underset{\sim}{V} \underset{\sim}{U} \phi \\
\stackrel{(20)}{=}-\underset{\sim}{V}(-i)^{\ell} \phi \\
=(-i)^{\ell+2}(\underset{\sim}{V} \phi) .
\end{gathered}
$$

Hence, $\underset{\sim}{V} \phi \in \mathcal{H}_{\ell+2}$, where $\ell+2$ is understood modulo 4 , and $\underset{\sim}{V} \operatorname{maps} \mathcal{H}_{\ell}$ onto $\mathcal{H}_{\ell+2}$ for $\ell=1,3$, if $\{\underset{\sim}{U}, \underset{\sim}{V}\}=0$ on $\mathcal{H}_{\text {odd }}$. This particular situation concerning the symmetries $\underset{\sim}{U}, \underset{\sim}{V}$ can be conveniently reformulated by using concepts $\tilde{\sim}$ from supersymmetry. This reformulation could also be useful to identify other examples that fit into the same scheme. In particular, we will embed $\underset{\sim}{U}$ and $\underset{\sim}{V}$ into a Lie superalgebra such that these symmetries "super-anticommute" in a sense to be explained. Since the spacial factors of $\underset{\sim}{U}$ and $\underset{\sim}{V}$, namely $\Pi$ and $\mathbb{\sim}$, commute anyway, it will suffice to consider their spin factors and hence to confine ourselves to finite-dimensional Lie superagebras.

Recall that a Lie superalgebra (LSA) is defined as a $\mathbb{Z}_{2}$ graded algebra. This means it is a linear space (over the field $\mathbb{R}$ or $\mathbb{C}$ ) of the form

$$
\mathcal{A}=\mathcal{A}_{0} \oplus \mathcal{A}_{1}
$$

equipped with a bilinear map

$$
[,\}: \mathcal{A} \times \mathcal{A} \rightarrow \mathcal{A}
$$

called the "super-bracket". An element $a \in \mathcal{A}_{0}$ or $a \in \mathcal{A}_{1}$ is called "homogeneous of degree $|a|$ " if $a \in \mathcal{A}_{|a|}$ and the following axioms (26)-(28) are understood to hold for homogeneous elements.

$$
\begin{aligned}
& |[a, b\}|=|a|+|b| \quad \bmod 2, \\
& \quad \text { "} \mathbb{Z}_{2}-\text { grading" } \\
& {[a, b\}=-(-1)^{|a||b|}[b, a\}} \\
& \quad \text { "(anti)symmetry" } \\
& {[a,[b, c\}\}=[[a, b\}, c\}+(-1)^{|a||b|}[b,[a, c\}\},} \\
& \text { "Jacobi identity" }
\end{aligned}
$$

see, e. g., [29].

In the following we will only use a special complex LSA defined as follows: Let $s$ be an (integer) spin quantum number and $\mathcal{M}_{0}$ denote the space of all complex $(2 s+1) \times(2 s+$ 1)-matrices. Let $\mathcal{M}_{1}$ be a copy of $\mathcal{M}_{0}$ such that

$$
\mathcal{M}=\mathcal{M}_{0} \oplus \mathcal{M}_{1}
$$

The matrices $M \in \mathcal{M}_{0}$ will be called "even" and those of $\mathcal{M}_{1}$ will be called "odd". The Lie superbracket [, \} is defined as the commutator $[A, B] \in \mathcal{M}_{0}$ for $A, B \in \mathcal{M}_{0}$, or, similarly, as $[A, B] \in \mathcal{M}_{1}$ for $A \in \mathcal{M}_{0}$ and $B \in \mathcal{M}_{1}$. On the other hand, the superbracket between two odd matrices $A, B \in \mathcal{M}_{1}$ is defined as the anti-commutator $\{A, B\} \in \mathcal{M}_{0}$. Finally, the superbracket is extended to $\mathcal{M}$ by means of bilinearity. It is straightforward to show that (26)-(28) is satisfied and hence $(\mathcal{M},[\}$,$) will be a complex LSA.$

We will denote by $\mathrm{U}$ and $\mathrm{V}$ the spin factors of $\underset{\sim}{U}$ and $\underset{\sim}{V}$, resp., that w.r.t. the eigenbasis $|m\rangle, m=-s, \ldots, s$, of $\underset{\sim}{s} z$ assume the form:

$$
\mathrm{U}=\left(\begin{array}{ccccccccc}
\ddots & 0 & 0 & 0 & 0 & 0 & 0 & 0 & 0 \\
0 & -i & 0 & 0 & 0 & 0 & 0 & 0 & 0 \\
0 & 0 & -1 & 0 & 0 & 0 & 0 & 0 & 0 \\
0 & 0 & 0 & i & 0 & 0 & 0 & 0 & 0 \\
0 & 0 & 0 & 0 & 1 & 0 & 0 & 0 & 0 \\
0 & 0 & 0 & 0 & 0 & -i & 0 & 0 & 0 \\
0 & 0 & 0 & 0 & 0 & 0 & -1 & 0 & 0 \\
0 & 0 & 0 & 0 & 0 & 0 & 0 & i & 0 \\
0 & 0 & 0 & 0 & 0 & 0 & 0 & 0 & \ddots
\end{array}\right)
$$


and

$$
\mathbf{V}=(-1)^{s}\left(\begin{array}{ccccccccc}
0 & 0 & 0 & 0 & 0 & 0 & 0 & 0 & . \cdot \\
0 & 0 & 0 & 0 & 0 & 0 & 0 & 1 & 0 \\
0 & 0 & 0 & 0 & 0 & 0 & 1 & 0 & 0 \\
0 & 0 & 0 & 0 & 0 & 1 & 0 & 0 & 0 \\
0 & 0 & 0 & 0 & 1 & 0 & 0 & 0 & 0 \\
0 & 0 & 0 & 1 & 0 & 0 & 0 & 0 & 0 \\
0 & 0 & 1 & 0 & 0 & 0 & 0 & 0 & 0 \\
0 & 1 & 0 & 0 & 0 & 0 & 0 & 0 & 0 \\
. & 0 & 0 & 0 & 0 & 0 & 0 & 0 & 0
\end{array}\right)
$$

Next, we split U and V into "even" and "odd" parts according to

$$
\begin{aligned}
U & =U_{0}+U_{1} \\
& =\left(\begin{array}{ccccccccc}
\ddots & 0 & 0 & 0 & 0 & 0 & 0 & 0 & 0 \\
0 & 0 & 0 & 0 & 0 & 0 & 0 & 0 & 0 \\
0 & 0 & -1 & 0 & 0 & 0 & 0 & 0 & 0 \\
0 & 0 & 0 & 0 & 0 & 0 & 0 & 0 & 0 \\
0 & 0 & 0 & 0 & 1 & 0 & 0 & 0 & 0 \\
0 & 0 & 0 & 0 & 0 & 0 & 0 & 0 & 0 \\
0 & 0 & 0 & 0 & 0 & 0 & -1 & 0 & 0 \\
0 & 0 & 0 & 0 & 0 & 0 & 0 & 0 & 0 \\
0 & 0 & 0 & 0 & 0 & 0 & 0 & 0 & \ddots
\end{array}\right) \\
& +\left(\begin{array}{ccccccccc}
\ddots & 0 & 0 & 0 & 0 & 0 & 0 & 0 & 0 \\
0 & -i & 0 & 0 & 0 & 0 & 0 & 0 & 0 \\
0 & 0 & 0 & 0 & 0 & 0 & 0 & 0 & 0 \\
0 & 0 & 0 & i & 0 & 0 & 0 & 0 & 0 \\
0 & 0 & 0 & 0 & 0 & 0 & 0 & 0 & 0 \\
0 & 0 & 0 & 0 & 0 & -i & 0 & 0 & 0 \\
0 & 0 & 0 & 0 & 0 & 0 & 0 & 0 & 0 \\
0 & 0 & 0 & 0 & 0 & 0 & 0 & i & 0 \\
0 & 0 & 0 & 0 & 0 & 0 & 0 & 0 & \ddots
\end{array}\right)
\end{aligned}
$$

and

$$
\begin{aligned}
& \mathrm{V}=\mathrm{V}_{0}+\mathrm{V}_{1} \\
& =(-1)^{s}\left(\begin{array}{ccccccccc}
0 & 0 & 0 & 0 & 0 & 0 & 0 & 0 & . \cdot \\
0 & 0 & 0 & 0 & 0 & 0 & 0 & 0 & 0 \\
0 & 0 & 0 & 0 & 0 & 0 & 1 & 0 & 0 \\
0 & 0 & 0 & 0 & 0 & 0 & 0 & 0 & 0 \\
0 & 0 & 0 & 0 & 1 & 0 & 0 & 0 & 0 \\
0 & 0 & 0 & 0 & 0 & 0 & 0 & 0 & 0 \\
0 & 0 & 1 & 0 & 0 & 0 & 0 & 0 & 0 \\
0 & 0 & 0 & 0 & 0 & 0 & 0 & 0 & 0 \\
. \cdot & 0 & 0 & 0 & 0 & 0 & 0 & 0 & 0
\end{array}\right) \\
& +(-1)^{s}\left(\begin{array}{ccccccccc}
0 & 0 & 0 & 0 & 0 & 0 & 0 & 0 & . \cdot \\
0 & 0 & 0 & 0 & 0 & 0 & 0 & 1 & 0 \\
0 & 0 & 0 & 0 & 0 & 0 & 0 & 0 & 0 \\
0 & 0 & 0 & 0 & 0 & 1 & 0 & 0 & 0 \\
0 & 0 & 0 & 0 & 0 & 0 & 0 & 0 & 0 \\
0 & 0 & 0 & 1 & 0 & 0 & 0 & 0 & 0 \\
0 & 0 & 0 & 0 & 0 & 0 & 0 & 0 & 0 \\
0 & 1 & 0 & 0 & 0 & 0 & 0 & 0 & 0 \\
. & 0 & 0 & 0 & 0 & 0 & 0 & 0 & 0
\end{array}\right)
\end{aligned}
$$

We embed these parts into $\mathcal{M}$ such that

$$
\mathrm{U}_{0}, \mathrm{~V}_{0} \in \mathcal{M}_{0} \text { and } \mathrm{U}_{1}, \mathrm{~V}_{1} \in \mathcal{M}_{1} \text {. }
$$

W.r.t. this embedding it can be easily shown that the following holds:

\section{Proposition 1}

$$
[\mathrm{U}, \mathrm{V}\}=0
$$

that is,

$$
\begin{aligned}
& {\left[\mathrm{U}_{0}, \mathrm{~V}_{0}\right]=\left[\mathrm{U}_{0}, \mathrm{~V}_{1}\right]=\left[\mathrm{V}_{0}, \mathrm{U}_{1}\right]=0,} \\
& \text { and }\left\{\mathrm{U}_{1}, \mathrm{~V}_{1}\right\}=0 .
\end{aligned}
$$

Thus, the general scenario where we can expect a similar alternating behaviour between level crossing and avoided level crossing as in the present paper is the case where there exist two super-commuting symmetries. Further aspects of super-symmetric quantum mechanics like the occurrence of super-symmetric pairs of Hamiltonians do not appear to be realized in the present case.

\section{A.2 Exact diagonalization for $s=1$}

The phenomenon of degenerate eigenspaces caused by super-symmetry occurs for all Hamiltonians of the form provided by Eqs. (4) and (8) in the main document, such that $B=0$ and hence ${ }_{\sim}$ Zeeman $=0$. The latter condition will be tacitly assumed in the remainder of this section, i. e., we always write

$$
\begin{aligned}
& \underset{\sim}{H}=\underset{\sim}{H} \mathrm{SI}+\underset{\sim}{H} \mathrm{HO} \\
& =D(\underset{\sim}{s} z)^{2} \otimes \underset{\sim}{\mathbb{1}}+\frac{\alpha}{2}\left\{\left({\underset{\sim}{s}}^{+}\right)^{2}+\left({\underset{\sim}{s}}^{-}\right)^{2}\right\} \otimes \underset{\sim}{x} \\
& +\underset{\sim}{\mathbb{1}} \otimes \omega\left(\stackrel{a}{\sim}^{\dagger} \underset{\sim}{\sim}+\frac{1}{2}\right)
\end{aligned}
$$

using $\left((\underset{\sim}{s} x)^{2}-(\underset{\sim}{s} y)^{2}\right)=\frac{1}{2}\left(\left({\underset{\sim}{s}}^{+}\right)^{2}+\left({\underset{\sim}{s}}^{-}\right)^{2}\right)$. Additionally, the question arises whether the ground state is degenerate, i.e., whether the ground state lies in one of the subspaces $\mathcal{H}_{1}$ or $\mathcal{H}_{3}$ and hence in both. Numerical evidence suggests that this will be the case for $D<0$ and odd $s$. In this section we will confirm this finding by exact diagonalization of the Hamiltonian for $s=1$ and $B=0$.

Since the Hamiltonian $H$ leaves the eigenspaces $\mathcal{H}_{\ell}$ of $U$ for $\ell=0,1,2,3$ invariant, it is possible to perform the diagonalization for each of the four subspaces separately. Due to the symmetry $\underset{\sim}{V}$ only one of the two cases $\mathcal{H}_{1}$ or $\mathcal{H}_{3}$ needs to be considered.

\section{A.2.1 Subspace $\mathcal{H}_{1}$}

The subspace $\mathcal{H}_{1}$ is spanned by the product states

$$
|m, n\rangle=\left|(-1)^{n+1}, n\right\rangle, \quad n=0,1,2, \ldots
$$

Let $\mathrm{H}$ denote the matrix of $H$ w. r. t. this basis. It is tridiagonal since $\underset{\sim}{x}$ is represented by a tri-diagonal matrix. The diagonal elements of $\mathrm{H}$ are obtained as

$$
\begin{aligned}
\mathrm{H}_{n n}= & \left\langle(-1)^{n+1}, n|\underset{\sim}{H}|(-1)^{n+1}, n\right\rangle \\
= & D\left\langle(-1)^{n+1}\left|(\underset{\sim}{s} z)^{2}\right|(-1)^{n+1}\right\rangle \\
& +\omega\left\langle n\left|\left({\underset{\sim}{a}}_{\sim}^{\dagger} a+\frac{1}{2}\right)\right| n\right\rangle
\end{aligned}
$$




$$
=D+\omega\left(n+\frac{1}{2}\right),
$$

since $\langle n|\underset{\sim}{x}| n\rangle=0$. For the upper secondary diagonal of $\mathrm{H}$ we have

$$
\begin{gathered}
\mathrm{H}_{n, n+1}=\left\langle(-1)^{n+1}, n|\underset{\sim}{H}|(-1)^{n}, n+1\right\rangle \\
=\frac{\alpha}{2}\left\langle(-1)^{n+1}\left|\left({\underset{\sim}{s}}^{+}\right)^{2}+\left({\underset{\sim}{s}}^{-}\right)^{2}\right|(-1)^{n}\right\rangle \times \\
\langle n|\underset{\sim}{x}| n+1\rangle \\
=\alpha \sqrt{2 \mu \omega n},
\end{gathered}
$$

since the secondary diagonal matrix elements of $\left(s_{z}\right)^{2}$ and $\underset{\sim}{H} \mathrm{HO}$ vanish and

$$
\left({\underset{\sim}{s}}^{+}\right)^{2}+(\underset{\sim}{s})^{-}=\left(\begin{array}{ccc}
0 & 0 & 2 \\
0 & 0 & 0 \\
2 & 0 & 0
\end{array}\right) .
$$

Obviously, $\mathrm{H}_{n+1, n}=\mathrm{H}_{n, n+1}$.

It follows that $\mathrm{H}$ is the same matrix as that of the operator

$$
\underset{\sim}{K} \equiv \underset{\sim}{H} \mathrm{HO}+D \underset{\sim}{\mathbb{1}}+\alpha \underset{\sim}{x}
$$

w. r. t. the harmonic oscillator eigenbasis $|n\rangle, n=0,1,2, \ldots$. Neglecting for the moment the constant energy shift due to $D \underset{\sim}{\mathbb{1}}$ we may write

$$
\begin{aligned}
& \underset{\sim}{H} \mathrm{HO}+\alpha \underset{\sim}{x}=\frac{1}{2 \mu}{\underset{\sim}{p}}^{2}+\frac{\mu \omega^{2}}{2}{\underset{\sim}{2}}^{2}+\alpha \underset{\sim}{x} \\
& =\frac{1}{2 \mu} p_{\sim}^{2}+\frac{\mu \omega^{2}}{2}\left(\underset{\sim}{x}+x_{0}\right)^{2}-\frac{\mu \omega^{2}}{2} x_{0}^{2},
\end{aligned}
$$

where

$$
x_{0} \equiv \frac{\alpha}{\mu \omega^{2}}
$$

We conclude that $\mathrm{K}$ is the matrix of a harmonic oscillator Hamiltonian with a spatially shifted minimum of the potential and a constant energy shift of

$$
\delta E=D-\frac{\mu \omega^{2}}{2} x_{0}^{2} \stackrel{(52)}{=} D-\frac{\alpha^{2}}{2 \mu \omega^{2}} .
$$

Its eigenvalues are hence of the form

$$
E_{n}^{(1)}=\omega\left(n+\frac{1}{2}\right)+D-\frac{\alpha^{2}}{2 \mu \omega^{2}},
$$

with the relative ground state energy

$$
E_{0}^{(1)}=\frac{\omega}{2}+D-\frac{\alpha^{2}}{2 \mu \omega^{2}} .
$$

Moreover, this result supports the remark in the main document referring to Eq. (15), since the ground state of the shifted oscillator and the ground state of the unshifted have a considerable overlap for small $x_{0}$.

\section{A.2.2 Subspace $\mathcal{H}_{0}$}

The subspace $\mathcal{H}_{0}$ is spanned by the product states

$$
|m, n\rangle=|0, n\rangle, n=0,2,4, \ldots
$$

Since the matrix elements of $\underset{\sim}{x}$ between different states of this basis vanish the matrix $\widetilde{\mathrm{K}}$ of the restriction of $\underset{\sim}{H}$ to the subspace $\mathcal{H}_{0}$ is already of diagonal form. Its diagonal elements that represent the energy eigenvalues read

$$
\begin{gathered}
\mathrm{K}_{n, n}=\left\langle 0, n\left|\mathbb{1} \otimes \underset{\sim}{H_{\mathrm{HO}}}+D(\underset{\sim}{s} z)^{2} \otimes \mathbb{1}\right| 0, n\right\rangle \\
=\omega\left(n+\frac{1}{2}\right),
\end{gathered}
$$

for $n=0,2,4, \ldots$, and yield the relative ground state energy

$$
E_{0}^{(0)}=\frac{\omega}{2}
$$

\section{A.2.3 Subspace $\mathcal{H}_{2}$}

Analogously to Sect. A.2.2, the subspace $\mathcal{H}_{2}$ is spanned by the product states

$$
|m, n\rangle=|0, n\rangle, n=1,3,5, \ldots
$$

Since the matrix elements of $\underset{\sim}{x}$ between different states of this basis vanish, the matrix $\widetilde{\mathrm{K}}$ of the restriction of $\underset{\sim}{H}$ to the subspace $\mathcal{H}_{0}$ is already of diagonal form. Its diagonal elements read

$$
\begin{aligned}
\mathrm{K}_{n, n}=\langle 0, n| \mathbb{1} \otimes \underset{\sim}{H} \mathrm{HO} & +D(\underset{\sim}{s} z)^{2} \otimes \mathbb{1}|0, n\rangle \\
& =\omega\left(n+\frac{1}{2}\right),
\end{aligned}
$$

for $n=1,3,5, \ldots$, and yield the relative ground state energy

$$
E_{1}^{(2)}=\frac{3 \omega}{2}\left(\stackrel{(59)}{>} E_{0}^{(0)} .\right.
$$

\section{A.2.4 Total ground state}

Summarizing the results of the Sects. A.2.1-A.2.3 we conclude that $E_{0}^{(1)}$ according to (55) represents the total ground state energy since

$$
E_{0}^{(1)}-E_{0}^{(0)}=D-\frac{\alpha^{2}}{2 m \omega^{2}}<0
$$

according to the assumption $D<0$ made in this section. This completes the arguments for the groundstate lying in the subspace $\mathcal{H}_{\text {odd }}$ in the case of $s=1$.

\section{A.3 Ground state for $s=2$}

According to numerical evidence, the groundstate lies in $\mathcal{H}_{\text {even }}$ for even $s$. We will confirm this result by rigorous estimates of the (relative) ground state energies for $s=2$. We again consider the Hamiltonian (40) and the various invariant subspaces $\mathcal{H}_{\ell}, \ell=0,1,2,3$. 


\section{A.3.1 Subspace $\mathcal{H}_{1}$}

The results of Subsection A.2.1 can largely be adopted, with the exception that for $s=2$ the matrix of $(\underset{\sim}{s})^{2}+(\underset{\sim}{s})^{2}$ assumes the form

$$
(\underset{\sim}{s})^{+}+(\underset{\sim}{s})^{2}=\left(\begin{array}{ccccc}
0 & 0 & 2 \sqrt{6} & 0 & 0 \\
0 & 0 & 0 & 6 & 0 \\
2 \sqrt{6} & 0 & 0 & 0 & 2 \sqrt{6} \\
0 & 6 & 0 & 0 & 0 \\
0 & 0 & 2 \sqrt{6} & 0 & 0
\end{array}\right) .
$$

By comparison with (48) this means that the parameter $\alpha$ in Sect. A.2.1 has to be replaced by $3 \alpha$ for the present case which gives the new expressions for the eigenvalues:

$$
E_{n}^{(1)}=\omega\left(n+\frac{1}{2}\right)+D-\frac{9 \alpha^{2}}{2 \mu \omega^{2}}, \quad \text { for } n=0,1,2 \ldots
$$

and the relative ground state energy

$$
E_{0}^{(1)}=\frac{\omega}{2}+D-\frac{9 \alpha^{2}}{2 \mu \omega^{2}} .
$$

\section{A.3.2 Subspace $\mathcal{H}_{2}$}

The subspace $\mathcal{H}_{2}$ is spanned by the product states

$$
|m, n\rangle=| \pm 2,0\rangle,|0,1\rangle,| \pm 2,2\rangle,|0,3\rangle, \ldots
$$

It can be further split into the two eigenspaces of $V$ formed by symmetric or antisymmetric linear combinations of the states $| \pm m, n\rangle$ in (68). These eigenspaces are also left invariant by the Hamiltonian $\underset{\sim}{H}$. In the following, we only consider the symmetric subspace $\mathcal{H}_{2, s}$ spanned by the states

$$
|\tilde{2}, 0\rangle,|0,1\rangle,|\tilde{2}, 2\rangle,|0,3\rangle, \ldots,
$$

where $|\tilde{2}\rangle$ denotes the spin state

$$
|\tilde{2}\rangle=\frac{1}{\sqrt{2}}(|2\rangle+|-2\rangle) .
$$

Let $\mathrm{K}$ denote the matrix of the Hamiltonian $\underset{\sim}{\mathrm{H}}$ w. r. t. the basis (69). Its diagonal entries read

$$
\begin{aligned}
\mathrm{K}_{n, n} & =\left\langle\tilde{2}, n\left|\mathbb{1} \otimes \underset{\sim}{H} H_{\mathrm{HO}}+D(\underset{\sim}{s} z)^{2} \otimes \mathbb{1}\right| \tilde{2}, n\right\rangle \\
& =\omega\left(n+\frac{1}{2}\right)+4 D, \quad \text { for even } n .
\end{aligned}
$$

Here we have used that the state $|\tilde{2}\rangle$ is an eigenstate of $(\underset{\sim}{s} z)^{2}$ corresponding to the eigenvalue $m^{2}=4$. Analogously,

$$
\begin{gathered}
\mathrm{K}_{n, n}=\left\langle 0, n\left|\mathbb{1} \otimes \underset{\sim}{H_{\mathrm{HO}}}+D(\underset{\sim}{s})^{2} \otimes \mathbb{1}\right| 0, n\right\rangle \\
=\omega\left(n+\frac{1}{2}\right), \quad \text { for odd } n,
\end{gathered}
$$

since the state $|0\rangle$ is an eigenstate of $(\underset{\sim}{s} z)^{2}$ corresponding to the eigenvalue $m^{2}=0$.

For the upper secondary diagonal entries of $\mathrm{K}$, we first consider the case of even $n$ and obtain

$$
\mathrm{K}_{n, n+1}=\frac{\alpha}{2}\left\langle 0, n\left|\left(\left({\underset{\sim}{s}}^{+}\right)^{2}+\left({\underset{\sim}{s}}^{-}\right)^{2}\right) \otimes \underset{\sim}{x}\right| \tilde{2}, n+1\right\rangle
$$

$$
\begin{aligned}
= & \frac{\alpha}{2 \sqrt{2}}\left(\left\langle 0\left|\left({\underset{\sim}{s}}^{+}\right)^{2}+\left({\underset{\sim}{s}}^{-}\right)^{2}\right| 2\right\rangle\right. \\
& \left.+\left\langle 0\left|\left({\underset{\sim}{s}}^{+}\right)^{2}+\left({\underset{\sim}{s}}^{-}\right)^{2}\right|-2\right\rangle\right)\langle n|\underset{\sim}{x}| n+1\rangle(7
\end{aligned}
$$

$$
\begin{aligned}
\stackrel{(65)}{=} & =\frac{\alpha}{2 \sqrt{2}}(2 \times 2 \sqrt{6}) \sqrt{2 \mu \omega n} \\
& =\alpha 2 \sqrt{3} \sqrt{2 \mu \omega n} .
\end{aligned}
$$

The result for odd $n$ is the same. It follows that, analogously to Sect. A.2.1, $\mathrm{K}$ equals the matrix of a shifted harmonic oscillator Hamiltonian $\underset{\sim}{\underset{\sim}{H}}$ plus a diagonal operator $\underset{\sim}{\Delta}$. Here,

$$
\underset{\sim}{\widehat{H}}=\frac{1}{2 \mu} p_{\sim}^{2}+\frac{\mu \omega}{2}\left(\underset{\sim}{x}+x_{0}\right)^{2}-\frac{\mu \omega}{2} x_{0}^{2}
$$

where

$$
x_{0}=\frac{2 \sqrt{3} \alpha}{\mu \omega^{2}},
$$

and hence

$$
\underset{\sim}{\widehat{H}}=\frac{1}{2 \mu} p_{\sim}^{2}+\frac{\mu \omega}{2}\left(\underset{\sim}{x}+x_{0}\right)^{2}-\frac{6 \alpha^{2}}{\mu \omega^{2}} .
$$

Further,

$$
\stackrel{\Delta}{\sim}=4 D \operatorname{diag}(1,0,1,0, \ldots,)
$$

We want to determine an upper bound of $E_{0}^{(2)}$ of the form

$$
E_{0}^{(2)} \leq\langle\Phi|\underset{\sim}{\underset{\sim}{H}}+\underset{\sim}{\Delta}| \Phi\rangle
$$

where $\Phi$ is chosen as the normalized ground state of $\underset{\sim}{\widehat{H}}$, to wit,

$$
\Phi(x)=\left(\frac{\mu \omega}{\pi}\right)^{1 / 4} \exp \left(-\frac{\mu \omega}{2}\left(x+x_{0}\right)^{2}\right) .
$$

Further, we will have to use the explicit form of the harmonic oscillator eigenfunctions

$$
\begin{aligned}
\phi_{n}(x)= & \left(2^{n} n ! \sqrt{\frac{\pi}{\mu \omega}}\right)^{-1 / 2} \\
& \exp \left(-\frac{\mu \omega}{2} x^{2}\right) H_{n}(\sqrt{\mu \omega} x),
\end{aligned}
$$

where $H_{n}(\ldots)$ denotes the $n$-th Hermite polynomial.

We first note that

$$
\langle\Phi|\underset{\sim}{\widehat{H}}| \Phi\rangle=\frac{\omega}{2}-\frac{6 \alpha^{2}}{\mu \omega^{2}} .
$$

Then we consider

$$
\langle\Phi \mid \Delta \sim \Phi\rangle=4 D \sum_{n=0,2, \ldots}\langle\Phi \mid n\rangle\langle n \mid \Phi\rangle
$$

After some calculations we obtain the intermediate result

$$
\begin{aligned}
\langle\Phi \mid n\rangle & =\int_{-\infty}^{\infty} \Phi(x) \phi_{n}(x) d x \\
& =\frac{\left(\sqrt{\mu \omega} x_{0}\right)^{n}}{\sqrt{2^{n} n !}} \exp \left(-\frac{\mu \omega x_{0}^{2}}{4}\right) .
\end{aligned}
$$


and hence

$$
\begin{aligned}
\langle\Phi|\Delta| \Phi\rangle & =4 D \sum_{n=0,2, \ldots}|\langle\Phi \mid n\rangle|^{2} \\
& =4 D \exp \left(-\frac{\mu \omega x_{0}^{2}}{2}\right) \cosh \left(\frac{\mu \omega x_{0}^{2}}{2}\right) \\
& =2 D\left(1+\exp \left(-\mu \omega x_{0}^{2}\right)\right) \\
& \stackrel{(79)}{=} 2 D\left(1+\exp \left(-\frac{12 \alpha^{2}}{\mu \omega^{3}}\right)\right) .
\end{aligned}
$$

Summarizing,

$$
\begin{aligned}
E_{0}^{(2)} \leq & \frac{\omega}{2}-\frac{6 \alpha^{2}}{\mu \omega^{2}} \\
& +2 D\left(1+\exp \left(-\frac{12 \alpha^{2}}{\mu \omega^{3}}\right)\right)
\end{aligned}
$$

\section{A.3.3 Total ground state}

Combining the previous results, we obtain

$$
\begin{aligned}
E_{0}^{(2)}-E_{0}^{(1)} \stackrel{(90,67)}{\leq} & -\frac{3}{2} \frac{\alpha^{2}}{\mu \omega^{2}} \\
& +D\left(1+2 \exp \left(-\frac{12 \alpha^{2}}{\mu \omega^{3}}\right)\right) \\
<\quad 0 &
\end{aligned}
$$

since $D<0$. This proves that $E_{0}^{(1)}$ cannot be the total ground state energy and hence the total ground state cannot lie in $\mathcal{H}_{\text {odd }}$. Actually, the numerical calculations show that it lies in the subspace $\mathcal{H}_{2, s}$ considered above, while the above analytical considerations prove only the weaker result that the total ground state lies in $\mathcal{H}_{\text {even }}$.

Finally, we would like to provide an example for our statement that the ground state with spin-phonon coupling consists mainly of the two ground states for $E=0$. The ground state in the discussed case of $s=2\left(D=-5, n_{\max }=1\right.$, $\alpha=0.5$, and $\omega=5$ in natural units) is

$$
\begin{aligned}
\left|\Phi_{0}\right\rangle= & +0.706684|m=2, n=0\rangle \\
& +0.706684|m=-2, n=0\rangle \\
& -0.034579|m=0, n=1\rangle .
\end{aligned}
$$

Thus, it contains only $0.1 \%$ admixture of other states.

\section{References}

1. D. Gatteschi, R. Sessoli, J. Villain, Molecular Nanomagnets. Mesoscopic Physics and Nanotechnology (Oxford, 2006)

2. S.J. Blundell, Contemp. Phys. 48, 275 (2007). https:// doi.org/10.1080/00107510801967415

3. J. Schnack, Contemp. Phys. 60, 127 (2019). https://doi. org/10.1080/00107514.2019.1615716

4. S.T. Liddle, J. van Slageren, Chem. Soc. Rev. 44, 6655 (2015). http://dx.doi.org/10.1039/C5CS00222B
5. R. Sessoli, D. Gatteschi, A. Caneschi, M.A. Novak, Nature 365, 141 (1993). https://doi.org/10.1038/ $365141 \mathrm{a} 0$

6. J.R. Friedman, M.P. Sarachik, J. Tejada, R. Ziolo, Phys. Rev. Lett. 76, 3830 (1996). https://doi.org/10.1103/ PhysRevLett.76.3830

7. L. Thomas, F. Lionti, R. Ballou, D. Gatteschi, R. Sessoli, B. Barbara, Nature 383, 145 (1996). https://doi.org/10. 1038/383145a0

8. F. Lionti, L. Thomas, R. Ballou, B. Barbara, A. Sulpice, R. Sessoli, D. Gatteschi, J. Appl. Phys. 81, 4608 (1997). https://doi.org/10.1063/1.365177

9. I. Chiorescu, W. Wernsdorfer, A. Müller, H. Bögge, B. Barbara, Phys. Rev. Lett. 84, 3454 (2000). https:// doi.org/10.1103/PhysRevLett.84.3454

10. W. Wernsdorfer, R. Sessoli, A. Caneschi, D. Gatteschi, A. Cornia, D. Mailly, J. Appl. Phys. 87, 5481 (2000). https://doi.org/10.1063/1.373379

11. D. Gatteschi, R. Sessoli, Angew. Chem. Int. Ed. 42, 268 (2003). https://doi.org/10.1002/anie.200390099

12. C.A.P. Goodwin, Dalton Trans. 49, 14320 (2020). https://doi.org/10.1039/D0DT01904F

13. C. Zener, Proc. R. Soc. Lond. Ser. A 137, 696 (1932). https://doi.org/10.1098/rspa.1932.0165

14. L.D. Landau, Phys. Z. Sowjetunion 2, 46 (1932)

15. E.C.G. Stueckelberg, Helv. Phys. Acta 5, 369 (1932). https://doi.org/10.5169/seals-110177

16. M.N. Leuenberger, D. Loss, Phys. Rev. B 61, 1286 (2000). https://doi.org/10.1103/PhysRevB.61.1286

17. K.N. Shrivastava, Phys. Rev. 187, 446 (1969). https:// doi.org/10.1103/PhysRev.187.446

18. K. Shrivastava, Phys. Lett. A 31, 454 (1970). http://www.sciencedirect.com/science/article/pii/ 0375960170903956

19. K. Shrivastava, Chem. Phys. Lett. 22, 622 (1973). http://www.sciencedirect.com/science/article/pii/ 0009261473870460

20. M. Vanhaelst, P. Matthys, E. Boesman, Phys. Stat. Sol. (b) 85, 639 (1978). https://onlinelibrary.wiley.com/doi/ abs/10.1002/pssb.2220850228

21. M.R. Pederson, N. Bernstein, J. Kortus, Phys. Rev. Lett. 89, 097202 (2002). https://link.aps.org/doi/10. 1103/PhysRevLett.89.097202

22. Y. Rechkemmer, F.D. Breitgoff, M. van der Meer, M. Atanasov, M. Hakl, M. Orlita, P. Neugebauer, F. Neese, B. Sarkar, J. van Slageren, Nat. Commun. 7, 10467 (2016). https://doi.org/10.1038/ncomms10467

23. L. Escalera-Moreno, N. Suaud, A. Gaita-Arino, E. Coronado, J. Phys. Chem. Lett. 8, 1695 (2017). https://doi. org/10.1021/acs.jpclett.7b00479

24. D.H. Moseley, S.E. Stavretis, K. Thirunavukkuarasu, M. Ozerov, Y. Cheng, L.L. Daemen, J. Ludwig, Z. Lu, D. Smirnov, C.M. Brown, A. Pandey, A.J. Ramirez-Cuesta, A.C. Lamb, M. Atanasov, E. Bill, F. Neese, Z.L. Xue, Nat. Commun. 9, 2572 (2018). https://doi.org/10.1038/ s41467-018-04896-0

25. F. Ortu, D. Reta, Y.S. Ding, C.A.P. Goodwin, M.P. Gregson, E.J.L. McInnes, R.E.P. Winpenny, Y.Z. Zheng, S.T. Liddle, D.P. Mills, N.F. Chilton, Dalton Trans. 48, 8541 (2019). http://dx.doi.org/10.1039/C9DT01655D

26. K. Irländer, J. Schnack, Phys. Rev. B 102, 054407 (2020). https://doi.org/10.1103/PhysRevB.102.054407

27. J. von Neumann, E.P. Wigner, Phys. Z. 30, 467 (1929) 
28. E.M. Chudnovsky, D.A. Garanin, R. Schilling, Phys. Rev. B 72(9), 094426 (2005). http://link.aps.org/ abstract/PRB/v72/e094426

29. V.G. Kac, Adv. Math. 26, 8 (1977). https://doi.org/10. 1016/0001-8708(77)90017-2
30. O. Agam, Nonequilibrium Effects in the Tunneling Conductance Spectra of Small Metallic Particles (Springer, New York, 1999), pp. 1205-1249 